\title{
ROLE OF ABERRANT GLYCOSYLATION IN OVARIAN CANCER DISSEMINATION
}

\author{
Razan Sheta and Dimcho Bachvarov \\ Department of Molecular Medicine, Laval University, Québec PQ, Canada \\ Centre de recherche du CHU de Québec, L'Hôtel-Dieu de Québec, Québec PQ, Canada
}

Epithelial ovarian cancer (EOC) is the most lethal gynecologic malignancy, and understanding the molecular changes associated with EOC etiology could lead to the identification of novel targets for more effective therapeutic interventions. Glycosylation represents a post-translational modification (PTM) of proteins playing a major role in various cellular functions. Moreover, glycosylation participates in major pathobiological events during tumor progression, as aberrant expression of glycan structures has been shown to contribute in alterations of specific cellular onco-phenotypes, including tumor cell proliferation, migration and invasion. This review aims to describe what is currently known about aberrant glycosylation in EOC, and more specifically, the contribution of aberrant O-linked glycosylation in EOC progression. We also discuss our findings about the altered GALNT3 overexpression in EOC and its involvement in disease dissemination through aberrant mucin O-glycosylation, as well as the potential to exploit the role of GALNT3 in understanding the general mechanisms of abnormal glycosylation implicated in EOC spreading. Further analyses in cancer glycobiology could significantly enhance our understanding of the molecular mechanisms of cancer progression, including EOC dissemination, and could lead to the identification of novel biomarkers/therapeutic targets for better management of this deadly disease. Biomed Rev 2014; 25: 83-92

Keywords: post-translational modification, N-linked glycosylation, O-linked glycosylation, GalNAc-transferases, ovarian cancer metastasis, mucins

\section{INTRODUCTION}

\section{Etiology of epithelial ovarian cancer}

Epithelial ovarian cancer (EOC) is a disease that is responsible for more cancer deaths among women in the Western world than all other gynecologic malignancies (1). EOC lethality primarily stems from the inability to detect the disease at an early, organ-confined stage, and the lack of effective therapies for advanced-stage disease. So there is urgent need for new therapeutic targets and a better understanding of the mechanisms involved in the spread of ovarian carcinoma. EOC is histologically classified into five different carcinoma subtypes, including low-grade serous, high-grade serous,

Received 8 December 2014, revised 22 December 2014, accepted 23 December 2014.

Correspondence to Dr Dimcho Bachvarov, Centre de recherche du CHU de Québec,

L'Hôtel-Dieu de Québec, 9 rue McMahon, Québec (Québec) Canada G1R 2J6. Tel.: +418 525 4444, ext. 15590,

Fax:+418 691 5439, E-mail: dimtcho.batchvarov@crhdq.ulaval.ca 
endometrioid, mucinous and clear cell (2); among these, serous carcinomas represent the most frequent type, comprising approximately $80 \%$ of all advanced EOC (2). Recent findings indicate that EOC subtypes are different diseases and suggest that future biomarker identification and clinical research studies should investigate each EOC subtype separately (3).

Treatment of EOC includes the combination of surgery and platinum based chemotherapy (4). These approaches, in addition to hormonal therapy, show up to $80 \%$ responsiveness in patients, but unfortunately more than $50 \%$ of EOC patients eventually relapse and will need additional treatments (4). Currently, there is no effective adjuvant treatment for EOC patients after surgery or chemotherapy.

It is well established that cancer invasion and metastasis represent the major cause of treatment failure. Approximately $70 \%$ of women with advanced-stage EOC have widespread intraperitoneal metastases, including the formation of malignant serous effusions within the peritoneal cavity $(1,2)$. Pleural effusions constitute the most frequent site of distant metastasis (FIGO stage IV disease). Unlike the majority of solid tumors, particularly at the primary site, cancer cells in effusions are not amenable to surgical removal, and failure in their eradication is one of the main causes of treatment failure. Indeed, despite advances in cytotoxic therapies (5, 6), only $30 \%$ of women with advanced-stage EOC survive 5 years after initial diagnosis (1). Thus, management of the metastatic disease becomes a crucial problem for the treatment of EOC. One possible way to resolve this problem is to target metastasis-specific pathways with novel therapies. Hence, focused identification of novel pro-metastatic mechanisms, target pathways and molecules could enhance our chances of discovering new and effective therapies.

Post-translational modifications (PTM) alter the threedimensional structures of proteins by covalently binding small molecules to them and therefore represent a major diversification mechanism for altering protein function. The main PTM types include protein covalent modifications such as phosphorylation, acetylation, glycosylation, methylation, and ubiquitination, and can be classified according to the type of the amino acid side chain modified, the category of the modifying enzyme, and the extent of reversibility (7). There are many documented studies investigating different PTMs and their association with cancer progression; among these, aberrant glycosylation has displayed rather important role in cancer progression $(8,9)$, including EOC dissemination (10).

\section{PROTEIN GLYCOSYLATION}

Glycosylation represents the most complex form of all PTM, and is defined by the regulated process of adding lipids and carbohydrates to proteins, as this regulation depends on various building enzymes and proteases that allow for the diversification of protein function. Many studies have advanced our understanding of the major roles of glycans, but no evidence has yet comprehensively explained their role or function under different normal and pathological conditions, which is mainly due to their enormous diversity. Glycans have been shown to play crucial roles in development, growth and the functionality of organisms (11). Cell surface glycans are mainly implicated in recognizing molecules that aid in the process of communication and adhesion (12). Analysis of the SWISS-PROT database showed that the majority of proteins of the sequon are glycosylated, as more than $50 \%$ of all proteins are characterized as glycoproteins (13). Glycosylation of proteins is relatively rare in prokaryotes and quite common in eukaryotes. Three types of glycosylation of proteins are currently known (including $C$-linked glycosylation, $\mathrm{N}$-linked glycosylation, and $O$-linked glycosylation), but $\mathrm{C}$-glycosylation, representing mannosylation of $\mathrm{C} 2$ of the indole ring of tryptophan residues is quite rare (14), as the major glycosylation types represent $\mathrm{N}$-linked and $\mathrm{O}$-linked (mucin) glycosylation.

\section{N-linked glycosylation}

$N$-linked-(or asparagine-type) glycosylation represents a fundamental and extensive PTM that results in the covalent attachment of an oligosaccharide onto asparagine residues of the polypeptide chains (15). The $N$-linked glycosylation of glycoproteins takes place in both prokaryotic and eukaryotic cells (15). Through the attachment of glycans at asparagine residues, $N$-linked glycosylation promotes protein folding by enhancing solubility and mediating interactions between nascent proteins and cellular proteins, such as chaperones. Three key processes in $\mathrm{N}$-linked glycosylation have been identified: (i) the lipid-mediated assembly of monosaccharides into glycans, which is performed by various enzymes in the endoplasmic reticulum (ER) - Golgi complex; (ii) the acceptance of a glycan by the consensus sequence Asn$\mathrm{X}-\mathrm{Ser} / \mathrm{Thr}$ (X for any amino acid except proline), and (iii) the oligosaccharyltransferase-catalyzed attachment of the glycan to the side chain of the asparagine residue (15). The initiation step of $\mathrm{N}$-glycan synthesis starts with the transfer of GlcNAc-P from UDP-GlcNAc to membrane-bound Dol-P to 
form GlcNAc-P-P-Dol, and this process is catalyzed by the enzyme GlcNAc-1-phosphotransferase (16). See Figure 1 for illustration of the principles of the $N$-glycosylation.

\section{O-linked (mucin) glycosylation}

$O$-glycosylation of proteins represents the most diverse PTM form. There are several types of $O$-glycosylation, and they have been characterized and differentiated based on their biosynthesis and linkage processes. These include: $O$-GalNAc or mucin-type, $O$-mannose, $O$-xylose, $O$-fucose, $O$-glucose, $O$-galactose and $O$-GlcNAc (12). The initiation process of
$O$-glycosylation, except for $O$-GalNAc, $O$-Xyl and $O$-GlcNAc, occurs in the ER. $O$-GalNAc initiation of proteins takes place in the Golgi (12) and the $O$-GlcNAc glycosylation takes place in the cytosol and the nucleus (17).

$O$-linked glycosylation mostly happens on serine and threonine residues (18), although as recently demonstrated, $O$-glycosylation can also be found on tyrosine residues (19). The initiation step of $O$-glycosylation is more complex than $\mathrm{N}$-glycosylation and involves the transfer of different monosaccharides to each of the six $O$-linked glycans. $O$-GalNAc glycosylation is more distinct as it involves two

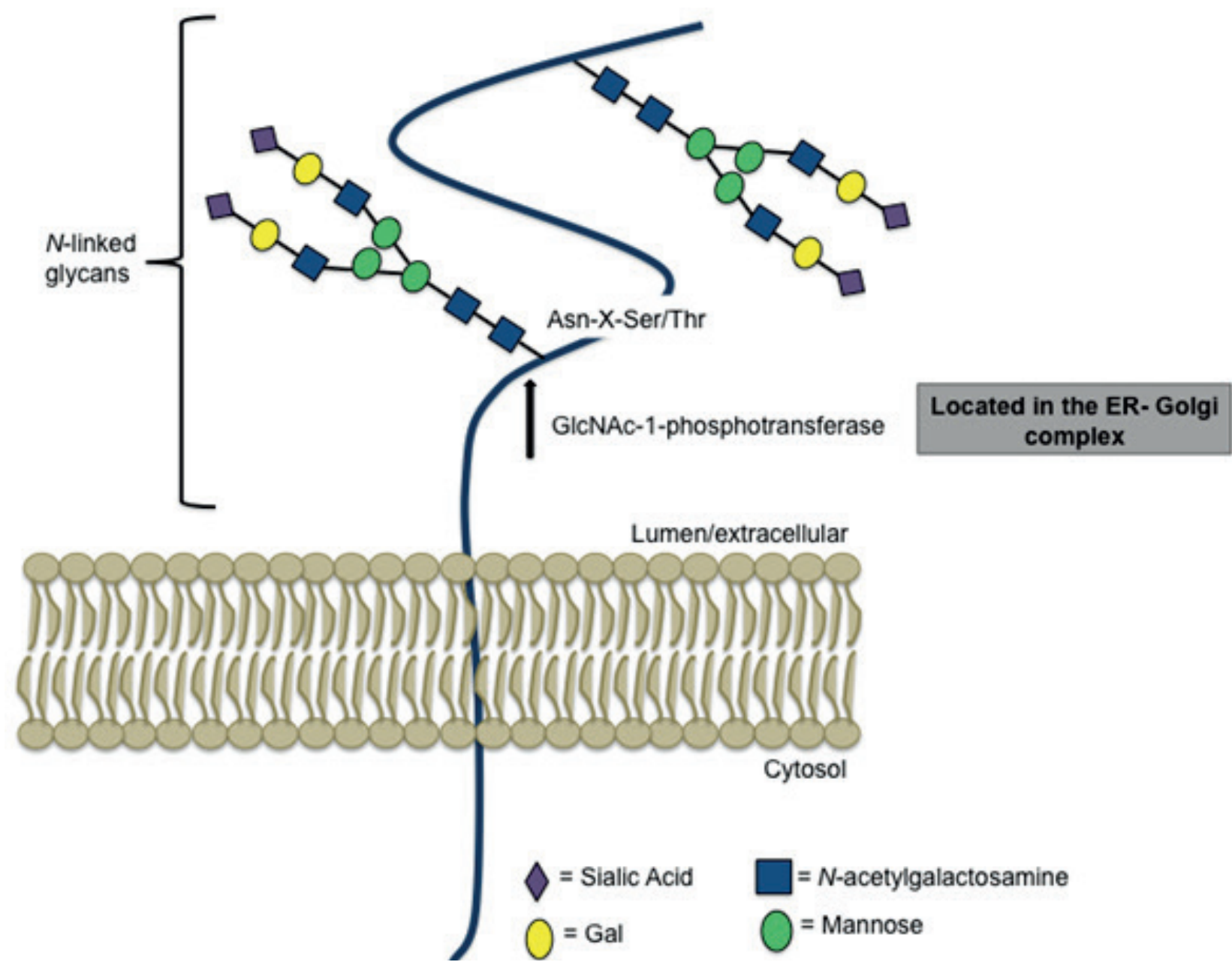

Figure 1. Schematic depiction of N-linked glycosylation. N-linked glycosylation is mainly linked to asparagine residues of proteins, specifically the Asn-X-Ser/Thr motif. N-linked glycosylation is initiated by en bloc transfer of an assembled complex glycan to the Asn residue. N-glycosylation biosynthesis starts with the high-mannose structure, followed by the branching of glycans and the addition of a variety of monosaccharides, this process is mainly dependent on the glycosyltransferases present, here we show the role of GlcNAc-1-phosphotransferase involved in the initiation process found in the ER-Golgi complex. The arrow indicates the transferase involved in the initiation process. The linkage of the first oligosaccharide is depicted with a (-). 
steps including the initiation step which is directed by 20 GalNAc-transferases, a family of enzymes known as the UDP$\mathrm{N}$-acetylgalactosamine: polypeptide $\mathrm{N}$-acetyl galactosaminyl transferases (GalNAcTs). Thus, the $O$-GalNAc initiation step involves the transfer of the monosaccharide GalNAc from UDP-GalNAc to the hydroxyl group of the serine, threonine or tyrosine residues found in the target protein substrate, which is followed by a processing step, where 30 or more glycosyltransferases create distinct glycan structures (20, 21). See Fig. 2 for illustration of the principles of GalNAc $O$-glycosylation.
As described above, cellular glycosylation mechanisms and their biosynthetic pathways are very complex and have shown to be fundamental to the changes in glycan processing and divergence. Numerous studies have shown that alterations in surface glycans are linked to disease progression and most importantly in cancer, as these alterations play pivotal roles in cancer initiation and progression $(22,23)$.

\section{ABERRANT GLYCOSYLATION IN CANCER}

Cancer cells have been characterized to undergo activation, rapid growth, adherence and invasion of neighboring cells/

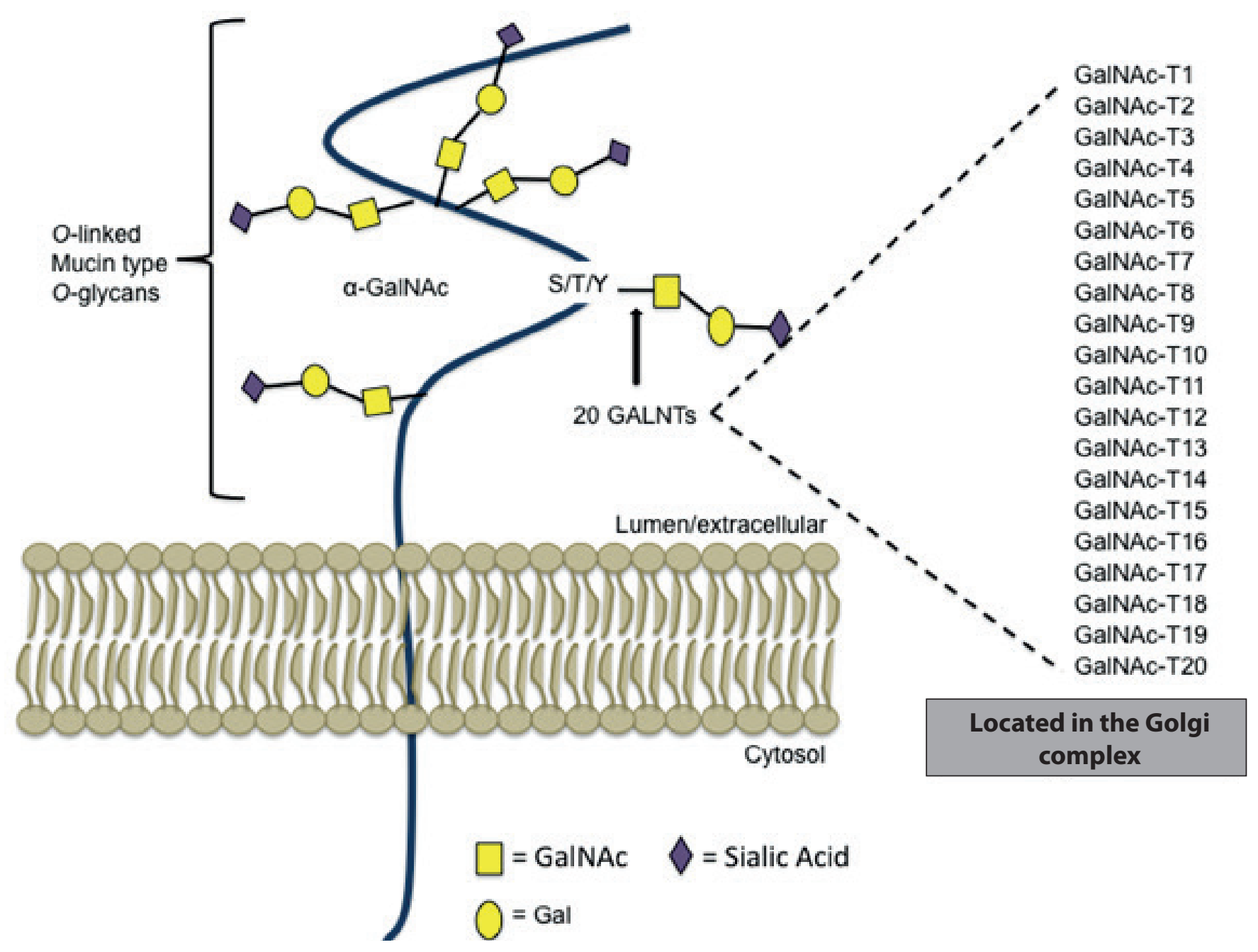

Figure 2. Schematic depiction of O-linked-(mucin type) glycosylation. O-linked glycosylation is mainly linked to Ser/Thr and Tyr residues. O-glycosylation is initiated by transfer of one of six monosaccharides; among these, the GalNAc-type-O-linked glycosylation pathway as depicted in the figure. GalNAc-type-O-linked glycosylation is uniquely controlled by the family of 20 polypeptide GalNAc-transferases located in the Golgi complex. The arrow indicates the genes involved in the initiation process. The linkage of the first oligosaccharide is depicted with a (-). 
tissues, and these processes are also linked to changes in the cell's glycosylation profiles (24). Studies have provided strong evidence for the existence of important link between abnormal glycosylation and tumorigenesis. Glycosylation changes characterized in cancer cells follow a variety of forms, as glycan alterations can be associated with loss or gain of expression, depending on the cell type and the specific glycan's structure. Aberrant glycosylation in cancer cells could affect certain ligand-receptor interactions and more importantly, could favor cancer cell proliferation, migration and invasion/metastasis.

Altered $N$-glycosylation of proteins has been reported in different cancer types, which has led to the identification of biomarkers for cancer prediction and diagnosis (25). Notably, the branching of $N$-glycans is involved in various biological functions including signal transduction, cell adhesion, proliferation, differentiation, and programmed cell death (26). The alteration of the branching alters the activity of relevant glycosyltransferases, thus affecting the regulation of various malignant processes, including cancer cells invasion and metastasis (26). A number of $N$-glycoproteins has been shown to be differentially expressed in different cancer types. Notably, differential glycosylation and increased expression of some $\mathrm{N}$-linked glycopeptides (including apolipoprotein B-100 and alpha-1-antichymotrypsin) has been identified in lung cancer serum pools compared to control pools (27). Some N-glycans, including ceruloplasmin, alpha-1 antichymotrypsin, and multimerin-1 were identified as biomarkers associated with hepatocellular carcinoma (HCC) (28). In addition, a study by Chen et al. (2011) found substantial concentration changes in $N$-linked glycoproteins between normal and HCC serum samples, as some of these glycoproteins included galectin-3 binding protein, insulinlike growth factor binding protein 3 , and thrombospondin 1 , previously found to be associated with HCC development (28). A strong overexpression of $\mathrm{N}$-glycoproteins, including cathepsin $\mathrm{L}$ and periostin (proteins, involved in extracellular matrix remodeling during metastasis) was shown to be associated with aggressive prostate cancer (30).

Similarly, $O$-glycans can play important role in cancerogenesis, as they could be in part responsible for the attachment, invasion and survival of cancer cells. Studies have demonstrated that $O$-glycan structures are truncated in many cancer types (31). Tumors associated with different mucin families show complex alterations in glycosylation profiles compared to normal cells. Recent studies showed that the downregulation of mucins contributes to the alterations observed in various cancer cell lines, which is associated with disease progression. $M U C 1$ is a glycoprotein integrated in the plasma membranes of epithelial cells as MUC1 has been repeatedly characterized as a major oncogene in different cancer types (32). Indeed, $M U C 1$ is aberrantly glycosylated and overexpressed in $>90 \%$ of breast carcinomas and in other cancer types of epithelial origin, including ovarian, lung, colon, and pancreatic carcinomas $(33,34)$. MUC4 has also been shown to be responsible for tumor progression (35). MUC4 expression has been associated with pancreatic cancer, as MUC4 is not expressed in normal pancreatic cells or tissue (35). MUC16 (or CA125) is now considered to be the most effective marker for early EOC detection and the monitoring of EOC reoccurrence after therapy (36).

The different members of the glycotransferase (GALNACTs) family were shown to be differentially expressed in malignant tumors compared to normal tissue. The deregulation in the expression of the different GALNACTs allows them to play diverse roles in cancerogenesis. Thus, GALNT1 is highly expressed in bladder cancer tissues, making it a potential prognostic marker in bladder cancer (37); GALNT2 is overexpressed in oral squamous cell carcinoma (38) and was also found to play a role in modifying EGFR glycosylation in HCC, which contributes to its malignant phenotype (39). GALNT3 is overexpressed in pancreatic cancer, and its suppression significantly correlates with pancreatic cancer cell growth inhibition (40). We have recently demonstrated that GALNT3 is strongly overexpressed in high grade serous EOC tumors, as compared to normal ovarian tissue (41). Upregulation of GALNT6 stabilizes $M U C 1$ protein by aberrant glycosylation in breast cancer cells, which allows the accumulation of the MUC1 protein in these cancer cells (42). Further, GALNT6 is expressed in gastric carcinomas and its expression was shown to associate with invasive disease (43). A role of GALNT7 in progression of cervical cancer (44) and human melanoma (45) has been demonstrated. GALNT9 expression was coupled with increased survival of neuroblastoma cancer cells making this enzyme a potential prognostic marker for this cancer type (46). GALNT10 was found to be overexpressed in gastric cancer, as its expression significantly correlated with the histological type and degree of this cancer type (47). Overexpression of GALNT11 was demonstrated in chronic lymphocytic leukemia (48). Mutations in the GALNT12 gene were linked to the development of colon 
cancer (49), and GALNT13 was specifically expressed in high-risk neuroblastoma (stage 4) patients, while no GALNT13 expression was detected in healthy individuals (50). GALNT14 contributes to the glycosylation of MUC13, which was observed to be significantly higher in EOC cells compared to their normal counterparts, suggesting a role of GALNT14 in EOC carcinogenesis, by directing aberrant MUC13 glycosylation (51). Moreover, a positive correlation was demonstrated between GALNT14 expression and the pro-apoptotic factor TRAIL in pancreatic cancer, indicative for a protective role of the GALNT14 enzyme in this cancer type (52).

\section{ALTERED GLYCOSYLATION IMPLICATED IN EOC ETIOLOGY}

\section{$N$-linked glycosylation in ovarian cancer}

$\mathrm{N}$-glycans play crucial roles that influence some of the glycoproteins properties and induce effects on the conformation, solubility, and recognition capacity of the proteins by glycanbinding proteins (16). Deficiencies in $\mathrm{N}$-glycan synthesis has lead to a variety of human diseases. Aberrant $N$-linked glycosylation has been also described and investigated in ovarian cancer. Several studies have examined this association; thus a number of $\mathrm{N}$-glycans displayed aberrant expression in different EOC subtypes, as mesothelin was shown to be overexpressed in high-grade serous, low-grade serous, and transitional-cell carcinomas, while versican and periostin were overexpressed in most subtypes of ovarian tumors (29). Gubbels et al. (2006) demonstrated that mesothelin-MUC16 binding is $\mathrm{N}$-glycan dependent, as this interaction was shown to facilitate EOC peritoneal dissemination (53). Another study by Zhang et al. (2014) investigated the association between $\mathrm{N}$-glycosylation alterations in glycoproteins secreted by EOC cells and metastasis of the disease. By using metabolic stable isotopic labeling, they have revealed a notable decrease in bisecting GlcNAc structure, as this decrease was found to be associated with a higher EOC metastatic potential (54). Interestingly, Anugraham et al have shown an expression of bisecting GlcNAc on $N$-linked glycoproteins, and this association was found to highly correlate with MGAT3 gene expression, a gene responsible for expressing the enzyme implicated in the synthesis of the core of the $N$-glycans (55). Their data demonstrated that definite $\mathrm{N}$-glycan substructures and their complexes are associated with specific epigenetic programming of synthetic enzymes in EOC (55). These studies suggest the involvement of $\mathrm{N}$-glycosylation in EOC spreading and metastasis, which further suggests the potential use of $\mathrm{N}$-glycans for biomarker development and targeted EOC therapy.

\section{O-linked (mucin-type) glycosylation in ovarian cancer}

Aberrant mucin-type $O$-glycosylation is one of the most abundant cancer-related PTMs, causing major pathological changes that in turn influence growth and survival of cancer cells and their ability for invasion and metastasis (31). Changes associated with the progression of EOC from primary to metastatic disease can affect the expression of certain tumorassociated antigens (TAA), subject to altered $O$-glycosylation. Thus, $M U C 1$ has been identified as a novel TAA, and several studies have demonstrated that $M U C 1$ is overexpressed and aberrantly $O$-glycosylated in most adenocarcinomas (34), in addition to being overexpressed on the cell surface of $90 \%$ of EOC tumors, including metastatic lesions (56). The major structural difference observed in tumor-associated MUC1 compared to a normal $M U C 1$ is that tumor $M U C 1$ contains shorter and less dense $O$-glycan chains, thus exposing more regions of the $M U C 1$ protein core (57). This feature of the tumorassociated $M U C 1$ makes it a possible EOC therapeutic target, which could delineate novel approaches for more effective EOC therapy. MUC13 represents another mucin associated with EOC dissemination. Indeed, $M U C 13$ has been overexpressed in EOC compared to normal tissue, and was shown to modulate various cellular functions such as cell adhesion and proliferation (58). A study by Wang et al. (2013) has provided evidence that GALNT14 contributes to the aberrant glycosylation of MUC13 in ovarian carcinogenesis (51). As mentioned above, MUC16 (CA125) is strongly associated with EOC pathobiology, as it currently represents the only clinically-recognized marker for EOC progression (59). A study by Robert et al has characterized the expression of several mucin genes in differnet EOC histological subtypes (60). They have shown that $M U C 1, M U C 2$ and $M U C 5 A C$ were overexpressed in EOC, while advanced disease was associated with decreased MUC3 and MUC4 levels (60). Moreover, other studies have revealed that ovarian cyst fluid can be a valuable source of mucins. It has been reported that MUC6 is a major component in ovarian cyst mucins (61) making it a good candidate to further elucidate the role of cyst mucins in tumorigenesis. In addition, $M U C 9$ was also found to be highly present in the serum of EOC patients (62).

Using an epigenomics approach, we have previously shown that DNA hypermethylation occurs in all (including less invasive/early) stages of ovarian tumorigenesis, while 
advanced disease was exclusively associated with DNA hypomethylation of a number of oncogenes, implicated in EOC progression, including invasion/metastasis (63). These experiments identified the GALNT3 gene as hypomethylated and overexpressed in high-grade EOC tumors, when compared to low-malignant potential EOC tumors and normal ovarian tissue (41). Additionally, the GALNT3 expression significantly correlated with shorter progressionfree survival intervals in women with advanced EOC (41). Consecutive short-hairpin (shRNA)-mediated GALNT3 knockdown experiments were strongly indicative for GALNT3 implication in EOC cell proliferation, migration, invasion and cell cycle control. Gene expression profiling and successive network and pathway analyses confirmed these findings, as numerous genes and pathways known previously to be implicated in EOC tumor invasion and metastasis were found to be downregulated upon GALNT3 suppression, while some tumor suppressor genes were induced (41). Moreover, GALNT3 knockdown resulted in considerable reduction of MUC1 protein in EOC cells, although the transcriptional level of MUC1 remained unchanged (41). A significantly diminished protein expression of other glycosylated proteins in EOC cells upon GALNT3 suppression was also observed, suggesting that GALNT3 may influence the posttranslational modification and stabilization of MUC1 and other $O$-glycosylated mucin-like targets in EOC cells (41). Also, the GALNT3 knockdown-mediated MUC1 suppression promoted the expression of the cell adhesion molecules E-cadherin and $\beta$-catenin, suggesting that the involvement of the GALNT3-MUC1 pathway in EOC invasion could include the destabilization of the E-cadherin/ $\beta$-catenin complex formation (41). Our data specify some of the mechanisms of abnormal $O$-glycosylation in ovarian carcinogenesis and the identification of the GALNT3 enzyme as EOC oncogene and novel EOC biomarker/possible molecular target for EOC therapy. These results warrant further and more profound studies of aberrant $O$-glycosylation in EOC.

\section{CONCLUSION AND PERSPECTIVES}

Glycosylation mediates various events associated with important biological expansion of the proteome. Aberrant glycosylation of glycan structures is now a new hallmark common of neoplastic transformation. Determining glycan structures is an essential step towards establishing a more comprehensive understanding of their roles in normal and pathological conditions, including cancer. In this review we focused on discussing the role of aberrant glycosylation in EOC. EOC is a highly metastatic disease and the leading cause of death from gynecologic malignancy. Hence, understanding the molecular changes associated with ovarian cancer metastasis could lead to the identification of targets for novel therapeutic interventions. Studies of disease-related alterations in glycan structures of glycoproteins can also lead to the discovery of new biomarkers for early EOC diagnostics and more efficient follow-up. Global analysis of proteins and their modifications has been majorly dependent on the recent advances in mass spectrometry tools for sequencing $N$ - and $O$ - glycans from cells and tissues. Progress in the field has developed strategies in performing both glycomic and glycoproteomic analysis. With these high-throughput technologies the dynamic area of $N$ - type and mucin-type $O$-glycosylation can be further explored and their role in tumorigenesis, including that of EOC, better understood. Moreover, the discovery of new and efficient inhibitors that can target glycosylation biosynthetic pathways could play important role in the understanding of specific/molecular mechanisms of normal and aberrant (disease-related) glycosylation, which undoubtedly will lead to the development of new and improved cancer treatment strategies, including more efficient EOC therapy.

\section{CONFLICT OF INTEREST}

The authors declare no conflict of interest.

\section{REFERENCES}

1. Siegel R, Ward E, Brawley O, Jemal, A. Cancer statistics. Cancer J Clinicians 2011; 61: 212-236. DOI:10.3322/ caac.20121.

2. Cho KR, Shih IM. Ovarian cancer. Annu Rev Pathol Mech Dis 2009; 4: 287-313. DOI:10.1146/annurev. pathol.4.110807.092246.

3. Brooks SA, Carter TM, Royle L, Harvey DJ, Fry SA, Kinch C, et al. Ovarian carcinoma subtypes are different diseases: implications for biomarker studies. PLos Med 2008; 5; e232. DOI:10.1371/journal.pmed.

4. Bhoola S, Hoskins WJ. Diagnosis and management of epithelial ovarian cancer. Obst Gynecol 2006; 107 : 1399-1410.

5. Fruscio R, Corso S, Ceppi L, Garavaglia D, Garbi A, Floriani I, et al. Conservative management of early-stage epithelial ovarian cancer: results of a large retrospective series. Ann Oncol 2012; 24: 138-144. DOI:10.1093/ annonc/mds241. 
6. Armstrong DK. Relapsed ovarian cancer: Challenges and management strategies for a chronic disease. Oncologist 2002; 7: 20-28. DOI:10.1634/theoncologist.7-suppl_5-20.

7. Walsh CT, Garneau-Tsodikova S, Gatto GJ. Protein posttranslational modifications: The chemistry of proteome diversifications. Angew Chem Int Ed 2005; 44: 7342-7372. DOI:10.1002/anie.200501023.

8. Bode AM, Dong Z. Post-translational modification of p53 in tumorigenesis. Natl Rev Cancer 2004; 4: 793-805. DOI: $10.1038 / \mathrm{nrc} 1455$

9. Karve TM, Cheema AK. Small changes huge impact: The role of protein posttranslational modifications in cellular homeostasis and disease. J Amino Acids 2011; 1-13. DOI:10.1042/BST0380137.

10. Sheehan KM, Calvert VS, Kay EW, Lu Y, Fishman D, Espina $\mathrm{V}$, et al. Use of reverse phase protein microarrays and reference standard development for molecular network analysis of metastatic ovarian carcinoma. Mol Cell Proteomics 2005; 4: 346-355. DOI:10.1074/mcp. T500003-MCP200

11. Varki A. Biological roles of oligosaccharides: all of the theories are correct. Glycobiology 1993; 3: 97-130. DOI:10.1093/glycob/3.2.97.

12. Brooks SA, Carter TM, Royle L, Harvey DJ, Fry $\mathrm{SA}$, Kinch $\mathrm{C}$, et al. Altered glycosylation of proteins in cancer: what is the potential for new anti-tumour strategies. Anticancer Agents Med Chem 2008; 8: 2-21. DOI:10.2174/187152008783330860.

13. Apweiler R, Hermjakob H, Sharon N. On the frequency of protein glycosylation, as deduced from analysis of the SWISS-PROT database. Biochim Biophys Acta General Subjects 1999; 1473: 4-8. DOI:10.1016/S03044165(99)00165-8.

14. Furmanek A, Hofsteenge J. Protein C-mannosylation: Facts and questions. Acta Biochim Pol 2000; 47: 781-789.

15. Schwarz F, Aebi M. Mechanisms and principles of N-linked protein glycosylation. Curr Opin Struct Biol 2011; 21: 576-582. DOI:10.1016/j.sbi.2011.08.005.

16. Schenk B, Fernandez F, Waechter CJ. The ins(ide) and outs(ide) of dolichyl phosphate biosynthesis and recycling in the endoplasmic reticulum. Glycobiology 2001; 11: 61R-70R. DOI:10.1093/glycob/11.5.61R.

17. Hu P, Shimoji S, Hart GW. Site-specific interplay between O-GlcNAcylation and phosphorylation in cellular regulation. FEBS Lett 2010; 584: 2526-2538. DOI:10.1016/j.febslet.2010.04.044
18. Stanley P. Golgi glycosylation. Cold Spring Harb Persp Biol 2011; 3: a005199-a005199. DOI:10.1101/ cshperspect.a005199.

19. Halim A, Brinkmalm G, Rüetschi U, WestmanBrinkmalm A, Portelius E, Zetterberg H, et al. Sitespecific characterization of threonine, serine, and tyrosine glycosylations of amyloid precursor protein/amyloid $\beta$-peptides in human cerebrospinal fluid. Proc Natl Acad Sci USA 2011; 108: 11848-11853.

20. Hagen, Ten KG. All in the family: the UDP-GalNAc: polypeptide N-acetylgalactosaminyltransferases. Glycobiology 2002; 13: 1R-16. DOI:10.1093/glycob/cwg007

21. Hang HC, Bertozzi CR. The chemistry and biology of mucin-type O-linked glycosylation. Bioorg Med Chem 2005; 13: 5021-5034. DOI:10.1016/j.bmc.2005.04.085.

22. Madsen CB, Petersen C, Lavrsen K, Harndahl M, Buus S, Clausen $\mathrm{H}$, et al. Cancer associated aberrant protein o-glycosylation can modify antigen processing and immune response. PLoS One 2012; 7: e50139. DOI:10.1371/journal.pone.0050139.s002.

23. Radhakrishnan P, Dabelsteen S, Madsen FB, Francavilla C, Kopp KL, Steentoft C, et al. Immature truncated O-glycophenotype of cancer directly induces oncogenic features. Proc Natl Acad Sci USA 2014; 111: E4066E4075. DOI:10.1073/pnas.1406619111.

24. Meany DL, Chan DW. Aberrant glycosylation associated with enzymes as cancer biomarkers. Clin Proteomics 2011; 8: 7. DOI:10.1186/1559-0275-8-7.

25. Tian Y, Zhang H. Characterization of disease-associated N-linked glycoproteins. Proteomics 2013; 13: 504-511. DOI: 10.1002/pmic.201200333.

26. Zhao Y, Sato Y, Isaji T, Fukuda T, Matsumoto A, Miyoshi $\mathrm{E}$, et al. Branched N-glycans regulate the biological functions of integrins and cadherins. FEBS J 2008; 275: 1939-1948. DOI:10.1111/j.1742-4658.2008.06346.x.

27. Zeng X, Hood BL, Sun M, Conrads TP, Day RS, Weissfeld $\mathrm{JL}$, et al. Lung cancer serum biomarker discovery using glycoprotein capture and liquid chromatography mass spectrometry. J Proteome Res 2010; 9: 6440-6449. DOI:10.1021/pr100696n.

28. Chen R, Tan Y, Wang M, Wang F, Yao Z, Dong L, et al. Development of glycoprotein capture-based label-free method for the high-throughput screening of differential glycoproteins in hepatocellular carcinoma. Mol Cell Proteomics 2011; 10: M110.006445-M110.006445. DOI:10.1074/mcp.M110.006445 
29. Tian Y, Yao Z, Roden RBS, Zhang H. Identification of glycoproteins associated with different histological subtypes of ovarian tumors using quantitative glycoproteomics. Proteomics 2011; 11: 4677-4687. DOI: $10.1002 /$ pmic.201000811

30. Tian Y, Bova GS, Zhang H. Quantitative glycoproteomic analysis of optimal cutting temperature-embedded frozen tissues identifying glycoproteins associated with aggressive prostate cancer. Anal Chem 2011; 83: 7013-7019. DOI: 10.1021/ac200815q

31. Brockhausen I. Pathways of O-glycan biosynthesis in cancer cells. Biochim Biophys Acta - General Subjects 1999; 1473: 67-95. DOI:10.1016/S0304-4165(99)00170-1.

32. Patton S, Gendler SJ, Spicer AP. The epithelial mucin, MUC 1, of milk, mammary gland and other tissues. Biochim Biophys Acta - General Subjects 1995; 1241: 407-424. DOI:10.1016/0304-4157(95)00014-3.

33. Singh R, Bandyopadhyay D. MUC1: A target molecule for cancer therapy. Cancer Biol Ther 2014; 6: 481-486. DOI:10.4161/cbt.6.4.4201.

34. Taylor-Papadimitriou J, Burchell J, Miles DW, Dalziel M. MUC1 and cancer. Biochim BiophysActa - General Subjects 1999; 1455: 301-313. DOI:10.1016/S09254439(99)00055-1.

35. Swartz MJ, Batra SK, Varshney GC, Hollingsworth MA, Yeo CJ, Cameron JL, et al. MUC4 expression increases progressively in pancreatic intraepithelial neoplasia. $\mathrm{Am}$ J Clin Pathol 2002; 117: 791-796. DOI:10.1309/7y7nm1wm-r0yk-m2va.

36. Gupta D, Lis CG. Role of CA125 in predicting ovarian cancer survival - a review of the epidemiological literature. JOvarian Res 2009; 2: 13. DOI:10.1186/17572215-2-13.

37. Ding M-X, Wang H-F, Wang J-S, Zhan H, Zuo Y-G., Yang D-L, et al. ppGalNAc T1 as a potential novel marker for human bladder cancer. Asian Pacific $J$ Cancer Preven 2012; 13: 5653-5657. DOI:10.7314/ APJCP.2012.13.11.5653.

38. Lin M-C, Huang M-J, Liu C-H, Yang T-L, Huang M-C. GALNT2 enhances migration and invasion of oral squamous cell carcinoma by regulating EGFR glycosylation and activity. Oral Oncol 2014; 50: 478484. DOI:10.1016/j.oraloncology.2014.02.003.

39. Wu YM, Liu CH, Hu RH, Huang MJ, Lee JJ, Chen CH, et al. Mucin glycosylating enzyme GALNT2 regulates the malignant character of hepatocellular carcinoma by modifying the EGF receptor. Cancer Res 2011; 71: 7270-7279. DOI:10.1158/0008-5472.CAN-11-1161.

40. Taniuchi K, Cerny RL, Tanouchi A, Kohno K, Kotani $\mathrm{N}$, Honke K, et al. Overexpression of GalNActransferase GalNAc-T3 promotes pancreatic cancer cell growth. Oncogene 2011; 30: 4843-4854. DOI:10.1038/ onc.2011.194.

41. Wang Z-Q, Bachvarova M, Morin C, Plante M, Gregoire J, Renaud M-C, et al. Role of the polypeptide $\mathrm{N}$-acetylgalactosaminyltransferase 3 in ovarian cancer progression: possible implications in abnormal mucin O-glycosylation. Oncotarget 2014; 5: 544-560.

42. Park JH, Nishidate T, Kijima K, Ohashi T, Takegawa K, Fujikane, T, et al. Critical roles of mucin 1 glycosylation by transactivated polypeptide $\mathrm{N}$-acetylgalactosaminyltransferase 6 in mammary carcinogenesis. Cancer Res 2010; 70: 2759-2769. DOI:10.1158/0008-5472.CAN-09-3911.

43. Gomes J, Marcos NT, Berois N, Osinaga E, Magalhaes A, Pinto-de-Sousa J, et al. Expression of UDP-N-acetyl-D-galactosamine: Polypeptide $\mathrm{N}$-acetylgalactosaminyltransferase- 6 in gastric mucosa, intestinal metaplasia, and gastric carcinoma. J Histochem Cytochem 2008; 57: 79-86. DOI:10.1369/ jhc.2008.952283.

44. Peng RQ, Wan HY, Li HF, Liu M, Li X, Tang H. MicroRNA-214 suppresses growth and invasiveness of cervical cancer cells by targeting UDP-N-acetyl- -D-galactosamine: Polypeptide N-Acetylgalactosaminyltransferase 7.J Biol Chem 2012; 287: 14301-14309. DOI:10.1074/jbc.M111.337642.

45. Gaziel-Sovran A, Segura MF, Di Micco R, Collins MK, Hanniford D, Vega-Saenz de Miera E, et al. miR-30b/30d regulation of GalNAc transferases enhances invasion and immunosuppression during metastasis. Cancer Cell 2011; 20: 104-118. DOI:10.1016/j.ccr.2011.05.027.

46. Berois N, Gattolliat CH, Barrios E, Capandeguy L, Douc-Rasy S, Valteau-Couanet D, et al. GALNT9 gene expression is a prognostic marker in neuroblastoma patients. Clin Chemistry 2013; 59: 225-233. DOI:10.1373/ clinchem.2012.192328.

47. Gao Y, Liu Z, Feng J, Sun Q, Zhang B, Zheng $\mathrm{W}$, et al. Expression pattern of polypeptide $\mathrm{N}$-acetylgalactosaminyltransferase-10 in gastric carcinoma. Oncol Lett 2012; 5: 113-116. DOI:10.3892/ ol.2012.980. 
48. Libisch MG, Casás M, Chiribao ML, Moreno P, Cayota A, Osinaga E, et al GALNT11 as a new molecular marker in chronic lymphocytic leukemia. Gene 2014; 533: 270-279. DOI:10.1016/j.gene.2013.09.052.

49. Gudaa K, Moinova H, He J, Jamison O, Ravi L, Natale $\mathrm{L}$, et al Inactivating germ-line and somatic mutationsin polypeptide $\mathrm{N}$-acetylgalactosaminyltransferase 12 in human colon cancers. Proc Natl Acad Sci USA 2009; 106: 12921-12925. DOI: 10.1073/pnas.0901454106.

50. Berois N. ppGalNAc-T13: A new molecular marker of bone marrow involvement in neuroblastoma. Clin Chem 2006; 52: 1701-1712. DOI:10.1373/ clinchem.2006.067975.

51. Wang R, Yu C, Zhao D, Wu M, Yang Z. The mucin-type glycosylating enzyme polypeptide $\mathrm{N}$-acetylgalactosaminyltransferase 14 promotes the migration of ovarian cancer by modifying mucin 13. Oncol Rep 2013; 30: 667-676. DOI: $10.3892 /$ or.2013.2493.

52. Holoch PA, Griffith TS. TNF-related apoptosis-inducing ligand (TRAIL): A new path to anti-cancer therapies. Eur J Pharmacol 2009: 625: 63-72. DOI: 10.1016/j. ejphar.2009.06.066.

53. Gubbels JAA, Belisle J, Onda M, Rancourt C, Migneault $\mathrm{M}$, Ho M, et al. Mesothelin-MUC16 binding is a high affinity, $\mathrm{N}$-glycan dependent interaction that facilitates peritoneal metastasis of ovarian tumors. Mol Cancer 2006; 5: 50. DOI:10.1186/1476-4598-5-50.

54. Zhang X, Wang Y, Qian Y, Wu X, Zhang Z, Liu X, et al. Discovery of specific metastasis-related n-glycan alterations in epithelial ovarian cancer based on quantitative glycomics. PLoS One 2014; 9: e87978. DOI:10.1371/journal.pone.0087978.s007.

55. Anugraham M, Jacob F, Nixdorf S, Everest-Dass AV, Heinzelmann-Schwarz V, Packer NH. Specific glycosylation of membrane proteins in epithelial ovarian cancer cell lines: Glycan structures reflect gene expression and DNA methylation status. Mol Cell Proteomics 2014; 13: 2213-2232. DOI:10.1074/mcp.M113.037085.
56. Wang L, Ma J, Liu F, Yu Q, Chu G, Perkins AC, et al. Expression of MUC1 in primary and metastatic human epithelial ovarian cancer and its therapeutic significance. Gynecol Oncol 2007; 105: 695-702. DOI:10.1016/j. ygyno.2007.02.004.

57. Price MR, Hudecz F, O’Sullivan C, Baldwin RW, Edwards PM, Tendler SJ. Immunological and structural features of the protein core of human polymorphic epithelial mucin. Mol Immunol 1990; 27: 795-802. DOI:10.1016/01615890(90)90089-I.

58. Chauhan SC, Vannatta K, Ebeling MC, Vinayek N, Watanabe A, Pandey KK, et al. Expression and functions of transmembrane mucin muc13 in ovarian cancer. Cancer Res 2009; 69: 765-774. DOI:10.1158/0008-5472. CAN-08-0587.

59. Guppy AE, Rustin GJ. CA125 response: Can it replace the traditional response criteria in ovarian cancer? Oncologist 2002; 7: 437-443. DOI:10.1634/theoncologist.7-5-437

60. Giuntoli RL 2nd, Rodriguez GC, Whitaker RS, Dodge R, Voynow JA. Mucin gene expression in ovarian cancers. Cancer Res 1998; 58: 5546-5550.

61. Lloyd KO, Yin BW, Tempst P, Erdjument-Bromage H. MUC-6 mucin is a major component of 'blood group substance' from human ovarian cyst fluid. Bioch Bioph Acta - General Subjects 2000; 1474: 410-414.

62. Maines-Bandiera S, Woo MM, Borugian M, Molday LL, Hii T, Gilks B, et al. Oviductal glycoprotein (OVGP1, MUC9), a differentiation-based mucin present in serum of women with ovarian cancer. Int J Gynecol Cancer 2010; 20: 16-22. DOI:10.1111/ IGC.0b013e3181bcc96d

63. Keita M, Wang ZQ, Pelletier JF, Bachvarova M, Plante $\mathrm{M}$, Gregoire J, et al. Global methylation profiling in serous ovarian cancer is indicative for distinct aberrant DNA methylation signatures associated with tumor aggressiveness and disease progression. Gynecol Oncol 2013; 128: 356-363. DOI:10.1016/j. ygyno.2012.11.036. 